\title{
Emergence of 1/3 magnetization plateau and successive magnetic transitions in Zintl phase $\operatorname{Eu}_{3} \operatorname{InAs}_{3}$
}

\author{
Ke Jia (贾可) $\odot,{ }^{1,2,3, *}$ Cui-Xiang Wang (王翠香) $\odot,{ }^{1,2,3, *}$ Xuejuan Dong (董雪娟), ${ }^{1}$ Nan Chen (陈楠), ${ }^{1,2}$ \\ Junzhuang Cong (丛君状), ${ }^{1}$ Guodong Li (李国栋) $\odot,{ }^{1,4, \dagger}$ Hai L. Feng (冯海) $\odot,{ }^{1, \dagger}$ Huaizhou Zhao (赵怀周) ${ }^{1,3,4}$ \\ and Youguo Shi (石友国) $)^{1,2,3,4,8}$ \\ ${ }^{1}$ Beijing National Laboratory for Condensed Matter Physics and Institute of Physics, Chinese Academy of Sciences, Beijing 100190, China \\ ${ }^{2}$ Center of Materials Science and Optoelectronics Engineering, University of Chinese Academy of Sciences, Beijing 100190, China \\ ${ }^{3}$ School of Physical Sciences, University of Chinese Academy of Sciences, Beijing 100190, China \\ ${ }^{4}$ Songshan Lake Materials Laboratory, Dongguan, Guangdong 523808, China
}

(Received 17 September 2021; accepted 23 November 2021; published 13 December 2021)

\begin{abstract}
Single crystals of $\mathrm{Eu}_{3} \mathrm{InAs}_{3}$ have been successfully synthesized by indium flux reactions. $\mathrm{Eu}_{3} \mathrm{InAs}_{3}$ crystallizes in the $\mathrm{Ca}_{3} \mathrm{AlAs}_{3}$-type structure with an orthorhombic space-group Pnma. Theoretical calculations indicate that $\mathrm{Eu}_{3} \mathrm{InAs}_{3}$ is a semiconductor with a direct band gap of $0.3 \mathrm{eV} . \mathrm{Eu}_{3} \mathrm{InAs}_{3}$ has large negative Seebeck coefficients in the range of 300-450 $\mu \mathrm{V} / \mathrm{K}$ at room temperature. $\mathrm{Eu}_{3} \mathrm{InAs}_{3}$ displays two antiferromagnetic transitions $\left(T_{N 1}=\right.$ 13 and $T_{N 2}=10 \mathrm{~K}$ ) for both $H \| b$ and $H \perp b$ which can be suppressed by magnetic fields. At $2 \mathrm{~K}$, field-induced ferromagnetic states are reached for both $H \| b$ and $H \perp b$. Particularly, the $H \| b$ magnetization curves show a plateau at $1 / 3$ of the saturated magnetization and an anomaly at $2 / 3$ of the saturated magnetization. $\mathrm{Eu}_{3} \operatorname{InAs}_{3}$ is a Zintl phase material showing a 1/3 magnetization plateau here.
\end{abstract}

DOI: 10.1103/PhysRevResearch.3.043178

\section{INTRODUCTION}

The classical Zintl phase was discovered in the 1930s as valence-specific compounds formed between electropositive elements, such as alkali metal or alkali-earth metal and the main groups 13-16 elements [1,2]. A large number of Zintl phase compounds have been reported [3-7], and they have been attracting great attention owing to their thermoelectric properties [8-15], magnetic properties [16-27], and superconductivity [28-30].

Recently, $M_{3} T \mathrm{Pn}_{3}$ Zintl phase compounds, where $M$ represents alkal- earth metal or europium, $T$ represents In or Al, and Pn represents As or P elements, show high Seebeck coefficients and have the potential for thermoelectric application. The antimonide series $\mathrm{Sr}_{3} \mathrm{GaSb}_{3}$ and $\mathrm{Ca}_{5} \mathrm{Al}_{2} \mathrm{Sb}_{6}$, for instance, bear high Seebeck coefficients above $300 \mu \mathrm{V} \mathrm{K}^{-1}[11,13]$. A maximum Seebeck coefficient of $500 \mu \mathrm{V} \mathrm{K}^{-1}$ has been observed in $\mathrm{Sr}_{3} \mathrm{AlSb}_{3}$ [8,12]. Recently, Eu $\mathrm{InAs}_{3}$ has been reported to have an unusually large negative Seebeck coefficient (absolute value $>400 \mu \mathrm{V} \mathrm{K}^{-1}$ at $700 \mathrm{~K}$ ) and a paramagnetic behavior down to $5 \mathrm{~K}$ [21]. Europium-containing Zintl

\footnotetext{
*These authors contributed equally to this work.

Corresponding author: gdli@iphy.ac.cn

¥Corresponding author: hai.feng@iphy.ac.cn

${ }^{\S}$ Corresponding author: ygshi@iphy.ac.cn
}

Published by the American Physical Society under the terms of the Creative Commons Attribution 4.0 International license. Further distribution of this work must maintain attribution to the author(s) and the published article's title, journal citation, and DOI. phase materials usually exhibit interesting magnetic behaviors [17-25]. For example, $\mathrm{Eu}_{3} \mathrm{Sn}_{2} \mathrm{P}_{4}$ and $\mathrm{Eu}_{14} \mathrm{InSb}_{11}$ are antiferromagnetically ordered $[18,19]$. EuAs 3 displays steplike behavior in the magnetization curves. $\mathrm{Eu}_{3} \mathrm{InP}_{3}$ has three successive magnetic orders and field-induced spin-flop transitions at low temperatures $[17,23]$. In comparison to other europiumcontaining Zintl phase materials displaying rich magnetic behaviors, a paramagnetism down to $5 \mathrm{~K}$ for $\mathrm{Eu}_{3} \mathrm{InAs}_{3}$ is unexpected. So, in this paper, we reinvestigate the structure, the Seebeck coefficient, and, particularly, the magnetic properties of $\mathrm{Eu}_{3} \mathrm{InAs}_{3}$. Our paper shows that $\mathrm{Eu}_{3} \mathrm{InAs}_{3}$ has large negative Seebeck coefficients similar to that reported in Ref. [21]. But, our magnetic measurements indicate that $\mathrm{Eu}_{3} \mathrm{InAs}_{3}$ shows successive magnetic orders and field-induced metamagnetic transitions, particularly, the magnetization curves show plateaus at $1 / 3$ of the saturation magnetization $\left(M_{\mathrm{S}}\right)$ and anomalies at $2 / 3$ of the $M_{\mathrm{S}}$. The phenomenon of the observation of fractional values $(1 / n)$ of the $M_{\mathrm{S}}$ is usually related to the geometric frustration resulting from triangular lattices or the competition of exchange interactions. $\mathrm{Eu}_{3} \mathrm{InAs}_{3}$ is a Zintl phase showing a $1 / 3$ magnetization plateau here.

\section{EXPERIMENT}

Single crystals of $\mathrm{Eu}_{3} \mathrm{InAs}_{3}$ were prepared by indium flux reactions. Elements Eu ( $>99.9 \%$, Alfa Aesar), In (4N, Alfa Aesar), and As (4N, Alfa Aesar) with a mole ratio of 1:30:1 were loaded into an alumina crucible. Then the crucible was placed into a silica tube. All these processes were carried out in an argon-filled glovebox to protect the elements. Then, the silica tube was transferred outside of the glovebox and sealed under dynamic vacuum using a $\mathrm{C}_{2} \mathrm{H}_{2} / \mathrm{O}_{2}$ torch, heated at $1100^{\circ}$ for $10 \mathrm{~h}$, then cooled slowly $\left(2^{\circ} / h\right)$ to $850^{\circ}$. After 
being dwelt for $5 \mathrm{~h}$ at $850^{\circ}$, the sealed tube was centrifuged immediately to remove the excess molten indium. Needleshaped single crystals of $\mathrm{Eu}_{3} \mathrm{InAs}_{3}$ up to several millimeters in length were obtained. It should be noted that different Eu:In:As mole ratios of 1:15:1 and 1:20:1 were also tried, but obtained $\mathrm{Eu}_{3} \mathrm{InAs}_{3}$ single crystals are relatively small. $\mathrm{Eu}_{3} \mathrm{InAs}_{3}$ is moderately air stable as previously reported [21] and can survive in the air for hours. The surface of $\mathrm{Eu}_{3} \mathrm{InAs}_{3}$ will turn black after being oxidated. We also tried to grow $\mathrm{La}_{3} \mathrm{InAs}_{3}$ using a similar method, however, the prepared samples are very sensitive to air, becoming yellowish powders after being exposed to air for a few minutes.

Single-crystal x-ray diffraction (SCXRD) was carried out on a Bruker D8 Venture diffractometer at $200 \mathrm{~K}$ using Mo $K \alpha$ radiation $(\lambda=0.71071 \AA)$. The single crystal was protected by flowing $\mathrm{N}_{2}$ gas during the measurement. A total of 1198 frames were collected. The frames were integrated with the Bruker SAINT software package using a narrow-frame algorithm. The integration of the data using an orthorhombic unit cell yielded a total of 22619 reflections to a maximum $\theta$ angle of $36.38^{\circ}(0.60-\AA$ resolution $)$ of which 2123 were independent (average redundancy 10.654, completeness $=$ $100.0 \%$, Rint $=8.16 \%, R \operatorname{sig}=3.77 \%)$ and $1739(81.91 \%)$ were greater than $2 \sigma(F 2)$. The final cell is based upon the refinement of the $X Y Z$ centroids of 4537 reflections above $20 \sigma(\mathrm{I})$ with $5.850^{\circ}<2 \theta<72.60^{\circ}$. Data were corrected for absorption effects using the multiscan method. The ratio of the minimum to the maximum apparent transmission was 0.626 . The crystal structure of $\mathrm{Eu}_{3} \mathrm{InAs}_{3}$ was solved and refined by analyzing the SCXRD data using the Bruker SHELXTL software package. The final anisotropic full-matrix least-squares refinement on $F 2$ with 43 variables converged at $R 1=2.73 \%$ for the observed data and $w R 2=4.88 \%$ for all data. The goodness of fit was 1.064, indicating the good quality of our sample and the result.

The composition of the single crystals was checked using energy dispersive $\mathrm{x}$-ray spectroscopy (EDX) operated in a Hitachi S-4800 scanning electron microscopy at an accelerating voltage of $15 \mathrm{kV}$ and an accumulation time of $90 \mathrm{~s}$.

First-principles calculations for $\mathrm{Eu}_{3} \mathrm{InAs}_{3}$ were performed using the Vienna ab initio simulation package. Spin-polarized calculations are conducted with the generalized gradient approximation and the Perdew-Burke-Ernzerhof exchangecorrelation function was adopted. The lattice constant is optimized until the energy difference is less than $10^{-7} \mathrm{eV}$, and the force convergence criteria is set to be $0.01 \mathrm{eV} / \AA$.

The temperature dependence of magnetic susceptibility $\chi(T)$ was measured in a superconducting quantum interference device VSM-7T (Quantum Design, Inc.) between 2 and $300 \mathrm{~K}$ with varied applied magnetic fields parallel (or vertical to the $b$ axis) of $\mathrm{Eu}_{3} \mathrm{InAs}_{3}$ crystals under field-cooled and zero-field-cooled conditions. Isothermal magnetization curves were measured at $2,10,20$, and $300 \mathrm{~K}$ with applied magnetic fields between -7 and $7 \mathrm{~T}$. The temperature dependence of specific-heat $C_{p}(T)$ was measured in a physical property measurement system-14 T, Quantum Design, Inc. from 2 to $300 \mathrm{~K}$ with varied magnetic fields parallel and vertical to the $b$ axis, respectively.

In the low-temperature range (4-350 K), the Seebeck coefficient and thermal conductivity of the $\mathrm{Eu}_{3} \mathrm{InAs}_{3}$ single crystal
TABLE I. Crystallographic data of $\mathrm{Eu}_{3} \mathrm{InAs}_{3}$.

\begin{tabular}{lc}
\hline \hline Chemical formula & $\mathrm{Eu}_{3} \mathrm{InAs}_{3}$ \\
Formula weight & $795.46 \mathrm{~g} / \mathrm{mol}$ \\
Radiation & Mo $K \alpha, 0.71073 \AA$ \\
Temperature & $200(2) \mathrm{K}$ \\
Crystal system & Orthorhombic \\
Space group & $P n m a$ \\
Unit-cell dimensions & $a=12.8973(4) \AA$ \\
& $b=4.39530(10) \AA$ \\
& $c=13.9273(5) \AA$ \\
Volume & $789.50(4) \AA^{3}$ \\
$Z$ & 4 \\
Density (calculated) & $6.692 \mathrm{~g} / \mathrm{cm}^{3}$ \\
Absorption coefficient & $38.753 \mathrm{~mm}^{-1}$ \\
$F(000)$ & 1348 \\
$\theta$ range for data collection & $2.15-36.38^{\circ}$ \\
Index ranges & $-21 \leqslant h \leqslant 21$ \\
& $-7 \leqslant k \leqslant 7$ \\
Independent reflections & $-23 \leqslant l \leqslant 22$ \\
Structure solution program & $2123[R($ int $)=0.0816]$ \\
Refinement program & $\mathrm{SHELXT} 2014 / 5$ \\
Function minimized & $\mathrm{SHELXT}-2018 / 3$ \\
Goodness of fin in $F^{2}$ & $\Sigma W\left(F_{o}^{2}-F_{c}^{2}\right) 2$ \\
$R 1\left(I>2 \sigma_{1}\right)$ & 1.064 \\
$\omega R 2\left(I>2 \sigma_{1}\right)$ & 0.0273 \\
$R 1$ (all data) & 0.0444 \\
$\omega R 2$ (all data) & 0.0436 \\
Weighting scheme & 0.0488 \\
& $1 /\left[\sigma^{2}\left(F_{o}^{2}\right)+(0.0146 P)^{2}+0.1060 P\right]$ \\
\hline \hline & where $P=\left(F_{\mathrm{o}}^{2}+2 F_{\mathrm{c}}^{2}\right)$ \\
\hline & \\
&
\end{tabular}

were measured in a homemade setup loaded in a liquid helium dewar. In the high-temperature range (300-750 K), the Seebeck coefficient was measured by CTA-3 (Cryoall).

\section{RESULTS AND DISCUSSION}

Crystal structure. The $\mathrm{SCXRD}$ data for $\mathrm{Eu}_{3} \mathrm{InAs}_{3}$ collected in this paper can be well refined with the orthorhombic spacegroup Pnma as reported by Rajput et al. [21]. $\mathrm{Eu}_{3} \mathrm{InAs}_{3}$ shares the same $\mathrm{Ca}_{3} \mathrm{AlAs}_{3}$-type structure with similar compounds $\mathrm{Eu}_{3} \mathrm{InP}_{3}$ and $\mathrm{Sr}_{3} \mathrm{InP}_{3}[6,16]$. The refined cell parameters, $a=$ 12.8973(4), $b=4.39530(10)$, and $c=13.9273(5) \AA$, are in good agreement with the values reported [21]. The detailed crystallographic data of $\mathrm{Eu}_{3} \mathrm{InAs}_{3}$ are summarized in Table I. The EDX results, Eu $43.20 \%$ (expected $42.86 \%$ ), In $14.33 \%$ (14.29\%), and As $42.27 \%$ (42.86\%) indicate a stoichiometric composition of $\mathrm{Eu}_{3} \mathrm{InAs}_{3}$. Atomic positions and temperature factors for $\mathrm{Eu}_{3} \mathrm{InAs}_{3}$ obtained from the refinements of the SCXRD data are summarized in Table II. The crystallographic information file can be found in the Supplemental Material [31].

The crystal structure of $\mathrm{Eu}_{3} \mathrm{InAs}_{3}$ is shown in Fig. 1(a). It can be described as long chains of $\operatorname{InAs}_{2} \mathrm{As}_{2 / 2}$ propagating along the $b$ direction, which are separated by $\mathrm{Eu}^{2+}$ ions. The linear $\operatorname{InAs}_{2} \mathrm{As}_{2 / 2}$ chains are composed of the corner-sharing $\mathrm{InAs}_{4}$ tetrahedral, and the $\mathrm{As}_{2 / 2}$ indicates two of the As atoms in $\mathrm{InAs}_{4}$ are shared with the neighbor-two $\mathrm{InAs}_{4}$ tetrahedral. In $\mathrm{Eu}_{3} \mathrm{InAs}_{3}, \mathrm{Eu}_{1}$ and $\mathrm{Eu}_{3}$ atoms are surrounded by six $\mathrm{As}$ 
TABLE II. Atomic coordinates and equivalent isotropic thermal parameters of $\mathrm{Eu}_{3} \mathrm{InAs}_{3}$.

\begin{tabular}{lcrcc}
\hline \hline & $x / a$ & $y / b$ & $z / c$ & $U($ eq. $)$ \\
\hline $\mathrm{Eu}_{1}$ & $0.64735(2)$ & 0.75 & $0.00136(2)$ & $0.00886(6)$ \\
$\mathrm{Eu}_{2}$ & $0.72755(2)$ & -0.25 & $0.29054(2)$ & $0.00821(6)$ \\
$\mathrm{Eu}_{3}$ & $0.56827(2)$ & 0.25 & $0.60819(2)$ & $0.00863(6)$ \\
$\mathrm{In}$ & $0.55605(3)$ & 0.25 & $0.20071(3)$ & $0.00744(8)$ \\
$\mathrm{As}_{1}$ & $0.60173(5)$ & 0.25 & $0.38858(5)$ & $0.00769(11)$ \\
$\mathrm{As}_{2}$ & $0.74879(5)$ & 0.25 & $0.12519(5)$ & $0.00772(11)$ \\
$\mathrm{As}_{3}$ & $0.45344(5)$ & -0.25 & $0.14214(5)$ & $0.00759(11)$ \\
\hline \hline
\end{tabular}

atoms, forming $\mathrm{EuAs}_{6}$ octahedra as shown in Fig. 1(b). $\mathrm{Eu}_{2}$ are surrounded by five As atoms, forming EuAs 5 pyramids. The corresponding bond distances obtained from the refinement of the SCXRD data are listed in Table III. The distances between Eu and As range from 3.0541 $\AA$ to $3.5972 \AA$, which are comparable to the values of 3.0653-3.6041 $\AA$ reported by Rajput et al. [21]. These data are also close to the ones reported for $\mathrm{Eu}_{3} \mathrm{InP}_{3}$ where the Eu-P bond lengths range from 2.989(2) to 3.644(2) ^ [16].

Electronic structure. The calculated band structure with spin-orbit coupling for $\mathrm{Eu}_{3} \mathrm{InAs}_{3}$ is shown in Fig. 2(a). It indicates that $\mathrm{Eu}_{3} \mathrm{InAs}_{3}$ is a semiconductor with a direct band gap of $0.3 \mathrm{eV}$. The effective mass at the $\Gamma$ point of the conduction band along different directions is calculated, the effective mass along $\Gamma-Y, \Gamma-X$, and $\Gamma-S$ is $0.743 m_{0}, 0.191 m_{0}$, and $0.207 m_{0}$, respectively. The effective masses of the valence band along the corresponding direction are $-0.752_{0}$, $-2.269 m_{0},-1.685 m_{0}$, where $m_{0}$ is the static mass of the electron. To better understand the electronic structure, the atom-projected partial density of states of $\mathrm{Eu}_{3} \mathrm{InAs}_{3}$ is plotted in Fig. 2(b). The states close to the valence-band maximum are mainly contributed from $\mathrm{As}$ and $\mathrm{Eu}$ atoms, indicating a certain degree of covalent bonding. Although the conduction band is mainly contributed by the Eu atoms.

Thermoelectric property. The temperature-dependent Seebeck coefficient for $\mathrm{Eu}_{3} \mathrm{InAs}_{3}$ measured between 170 and $750 \mathrm{~K}$ is shown in Fig. 3(a). Negative Seebeck coefficient values are observed for the whole temperature range measured, indicating the charge carriers are dominantly electrons. The $\mathrm{Eu}_{3} \mathrm{InAs}_{3}$ displays large Seebeck coefficients between -270 and $-500 \mu \mathrm{V} \mathrm{K}^{-1}$ from room temperature to $750 \mathrm{~K}$. It should

(a)
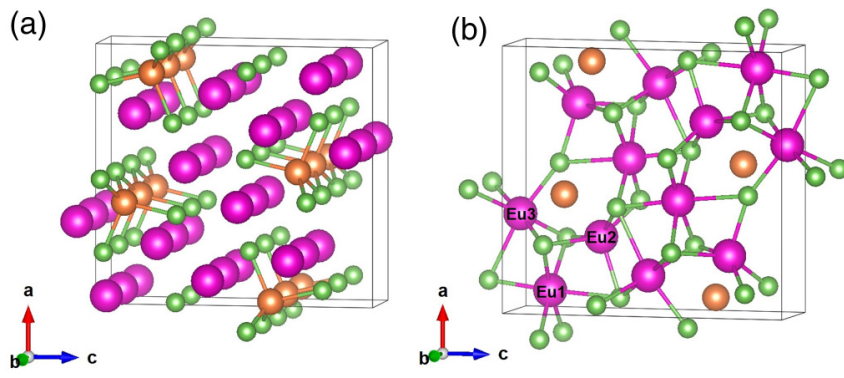

FIG. 1. Crystal structure of $\mathrm{Eu}_{3} \mathrm{InAs}_{3}$ showing the (a) $\operatorname{InAs}_{2} \mathrm{As}_{2 / 2}$ chains and (b) Eu-As bonds. Purple, orange, and green balls represent the $\mathrm{Eu}, \mathrm{In}$, and As atoms, respectively. The structure is tripled along the $b$ axis in (a).
TABLE III. Selected bond lengths in $\mathrm{Eu}_{3} \mathrm{InAs}_{3}$.

\begin{tabular}{lc}
\hline \hline Bond & Bond length $(\AA)$ \\
\hline$E u_{1}-\mathrm{As}_{1}$ & $3.5972(7)$ \\
$\mathrm{Eu}_{1}-\mathrm{As}_{2}$ & $3.0847(5)$ \\
$\mathrm{Eu}_{1}-\mathrm{As}_{2}$ & $3.0847(5)$ \\
$\mathrm{Eu}_{1}-\mathrm{As}_{3}$ & $3.2424(5)$ \\
$\mathrm{Eu}_{1}-\mathrm{As}_{3}$ & $3.2424(5)$ \\
$\mathrm{Eu}_{1}-\mathrm{As}_{3}$ & $3.1779(7)$ \\
$\mathrm{Eu}_{2}-\mathrm{As}_{1}$ & $3.0541(5)$ \\
$\mathrm{Eu}_{2}-\mathrm{As}_{1}$ & $3.0541(5)$ \\
$\mathrm{Eu}_{2}-\mathrm{As}_{2}$ & $3.1950(5)$ \\
$\mathrm{Eu}_{2}-\mathrm{As}_{2}$ & $3.1950(5)$ \\
$\mathrm{Eu}_{2}-\mathrm{As}_{3}$ & $3.0605(7)$ \\
$\mathrm{Eu}_{3}-\mathrm{As}_{1}$ & $3.0889(7)$ \\
$\mathrm{Eu}_{3}-\mathrm{As}_{1}$ & $3.1046(5)$ \\
$\mathrm{Eu}_{3}-\mathrm{As}_{1}$ & $3.1046(5)$ \\
$\mathrm{Eu}_{3}-\mathrm{As}_{2}$ & $3.2331(5)$ \\
$\mathrm{Eu}_{3}-\mathrm{As}_{2}$ & $3.2331(5)$ \\
$\mathrm{Eu}_{3}-\mathrm{As}_{3}$ & $3.4885(7)$ \\
\hline \hline
\end{tabular}

be noted that the measured Seebeck coefficients for $\mathrm{Eu}_{3} \mathrm{InAs}_{3}$ are sample dependent as displayed in Fig. 3(a). These large negative Seebeck coefficients are comparable to the values of -310 to $-430 \mu \mathrm{V} \mathrm{K}^{-1}$ in the temperature range from 300 to $700 \mathrm{~K}$ reported by Rajput et al. [21]. Both samples S1 and S2 show bumps around $420 \mathrm{~K}$. This behavior has also been reported by Rajput et al., and it is possibly due to a small amount of residual In flux which has a melting point of $430 \mathrm{~K}$ [21]. We tried to measure the resistivity of $\mathrm{Eu}_{3} \mathrm{InAs}_{3}$ samples from room temperature to low temperatures and found that the resistivity was too large to measure, indicating the as-grown $\mathrm{Eu}_{3} \mathrm{InAs}_{3}$ single crystals have very low carrier concentration. The temperature-dependent thermal conductivity was measured in the temperature range of $4.2-350 \mathrm{~K}$ as shown in Fig. 3(b). Since the $\mathrm{Eu}_{3} \mathrm{InAs}_{3}$ sample has very low electrical conductivity, the electronic contribution of thermal conductivity can be reasonably ignored. The thermal conductivity result of Fig. 3(b) could be regarded as mainly dominated by the lattice contribution. As the increasing of temperature, the thermal conductivity undergoes a typical first increasing to a peak value of $35.8 \mathrm{~W} \mathrm{~K}^{-1} \mathrm{~m}^{-1}$ and then decreasing to an asymptotic high-temperature value of $4.0 \mathrm{~W} \mathrm{~K}^{-1} \mathrm{~m}^{-1}$. The decreasing of thermal conductivity in the high-temperature range is mainly due to the phonon-phonon umklapping scattering.

Magnetism. Temperature-dependent magnetic susceptibility curves $\chi(T)$ of $\mathrm{Eu}_{3} \mathrm{InAs}_{3}$, measured at varied applied magnetic fields $(H \| b)$ are shown in Figs. 4(a) and 4(b). The $\chi(T)$ curve measured under 1 kOe shows two kinks at 13 and $10 \mathrm{~K}$, respectively, indicating possible successive antiferromagnetic (AFM) transitions. The two AFM transitions are further confirmed from the temperature-dependent specific-heat curve $C_{p}(T)$, measured without a magnetic field [see Fig. 5(a)], which displays two $\lambda$-type anomalies evolving at $13 \mathrm{~K}$ (noted as $T_{N 1 \|}$ ) and $10 \mathrm{~K}$ (noted as $\left.T_{N 2 \|}\right)$, respectively. When the magnetic fields increase up to $10 \mathrm{kOe}$, the $T_{N 2 \|}$ shifts to lower temperatures with increasing of the magnetic fields as shown in Fig. 4(a), indicating that the magnetic fields 

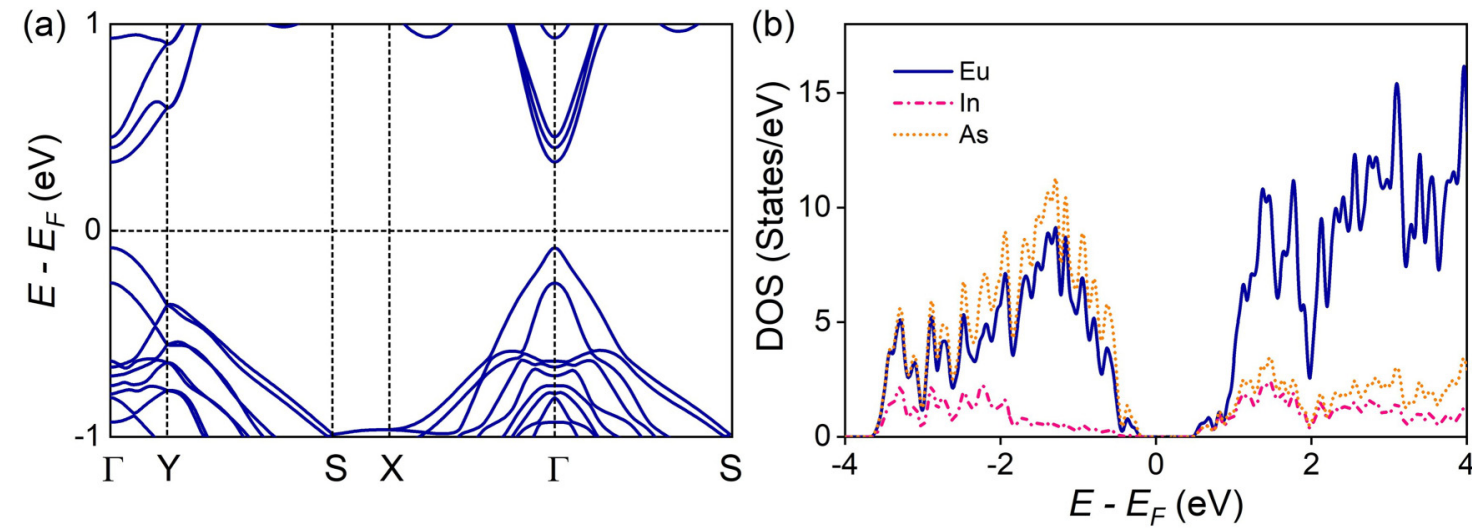

FIG. 2. (a) The band structure of $\mathrm{Eu}_{3} \mathrm{InAs}_{3}$ with spin-orbit coupling; (b) atom-projected density of states of $\mathrm{Eu}_{3} \mathrm{InAs} \mathrm{s}_{3}$.

suppress the second AFM order. The second AFM transition disappeared, and the first AFM transition is suppressed to $T_{N 1 \|}=11 \mathrm{~K}$ when the magnetic fields are $20 \mathrm{kOe}$ [see Fig. 4(b)], which is also confirmed from the $C_{p}(T)$ curves measured at $20 \mathrm{kOe}$ showing only one $\lambda$-type anomaly around $11 \mathrm{~K}$ [see Fig. 5(a)]. The $T_{N 1 \|}$ is further suppressed with increasing magnetic fields and disappears when the magnetic fields are at 50 and $70 \mathrm{kOe}$ as shown in Fig. 4(b). The suppression of AFM order can also be seen from $C_{p}(T)$ curves shown in Fig. 5(a) that a $\lambda$-type anomaly is around $7 \mathrm{~K}$ when measured at $30 \mathrm{kOe}$ and disappeared when measured at 50 kOe. The $\chi(T)$ curves measured at 50 and 70 kOe show ferromagneticlike behavior. The Curie temperatures $T_{\mathrm{c}}$, determined from the corresponding $d \chi / d T$ curves are 17 and $20 \mathrm{~K}$ for the curves measured at 50 and $70 \mathrm{kOe}$, respectively.

The $\chi(T)$ curves of $\mathrm{Eu}_{3} \mathrm{InAs}_{3}$ measured at varied magnetic fields $(H \perp b)$ are shown in Fig. 4(c). The $\chi(T)$ curve shows two kinks at 10 and $13 \mathrm{~K}$ under $H=1 \mathrm{kOe}$, which is similar to the $H \| b$ data and indicate two AFM transitions. The two AFM transitions are confirmed by the data of the $C_{p}(T)$ curves measured without a field, showing two $\lambda$-type anomalies around $13\left(T_{N 1 \perp}\right)$ and $10 \mathrm{~K}\left(T_{N 2 \perp}\right)$, respectively [see Fig. 5(c)]. When the magnetic fields increase up to 10 $\mathrm{kOe}$, the $\left(T_{N 2 \perp}\right)$ moves toward lower temperatures, $10.5 \mathrm{~K}$ for $H=5 \mathrm{kOe}$ and $9.5 \mathrm{~K}$ for $H=10 \mathrm{kOe}$. The $\left(T_{N 2 \perp}\right)$ disappears and the $\left(T_{N 1 \perp}\right)$ shifts to a lower temperature of 12.5
$\mathrm{K}$ when the magnetic field is $20 \mathrm{kOe}$. Correspondingly, the $C_{p}(T)$ curve measured under 20 kOe shows only one anomaly around $12.5 \mathrm{~K}$ [see Fig. 5(c)]. The $\left(T_{N 1 \perp}\right)$ shifts to a lower temperature of $11 \mathrm{~K}$ with $H=30 \mathrm{kOe}$, which can be seen from both the $\chi(T)$ and the $C_{p}(T)$ curves measured under $30 \mathrm{kOe}$. When $H$ is 50 and $70 \mathrm{kOe}$, the $\chi(T)$ curves show ferromagneticlike behavior, and the $T_{\mathrm{cs}}$ are 15 and $17 \mathrm{~K}$, respectively, as determined from the corresponding $d \chi / d T$ data.

Figure 4(d) displays the $\chi^{-1}(T)$ curves of $\mathrm{Eu}_{3} \mathrm{InAs}_{3}$ measured under a magnetic field of $10 \mathrm{kOe}$ with $H \| b$ and $H \perp b$, respectively. Fitting the 50 - to $300-\mathrm{K}$ data with CurieWeiss law, $\chi=C /(T-\theta)$, where $C$ and $\theta_{\mathrm{W}}$ are the Curie constant and the Weiss temperature, respectively, values of $C=24.34 \mathrm{emu} \mathrm{K} \mathrm{mol}^{-1}$ and $\theta_{\mathrm{W}}=16 \mathrm{~K}$ were obtained for $H \| B$ and $C=25.45 \mathrm{emu} \mathrm{K} \mathrm{mol}^{-1}$ and $\theta_{\mathrm{W}}=17 \mathrm{~K}$ for $H \perp b$. According to the $C$ values, effective moment $\mu_{\text {eff }}$ of 8.06 and $8.24 \mu_{B} / \mathrm{Eu}^{2+}$ were obtained for $H \| B$ and $H \perp b$, respectively, which are close to the theoretical value of $7.94 \mu_{B}$ for $\mathrm{Eu}^{2+}(S=7 / 2)$ and are comparable with the ones reported for other $\mathrm{Eu}^{2+}$ compounds $\mathrm{Eu}_{3} \mathrm{InP}_{3}, \mathrm{Eu}_{3} \mathrm{GaAs}_{3}$, and $\mathrm{Eu}_{3} \mathrm{AlAs}_{3}$ [27].

To further study the field-induced magnetic states in $\mathrm{Eu}_{3} \mathrm{InAs}_{3}$, isothermal magnetization curves $M(H)$ have been measured at 2, 10, 20, and $300 \mathrm{~K}$ with $H \| b$ [see Fig. 6(a)]. At $2 \mathrm{~K}$, the magnetization of $\mathrm{Eu}_{3} \mathrm{InAs}_{3}$ shows a sharp increase
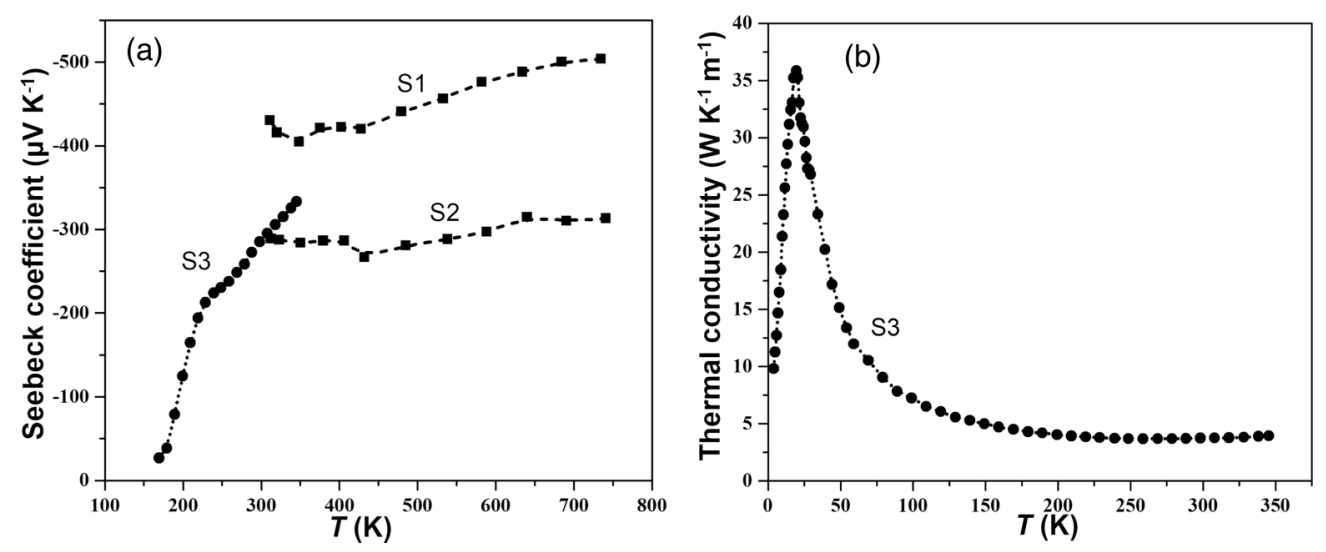

FIG. 3. (a) Temperature-dependent Seebeck coefficient and (b) thermal conductivity for $\mathrm{Eu}_{3} \mathrm{InAs} \mathrm{s}_{3}$. S1, S2, and S3 indicate three different single crystals. 

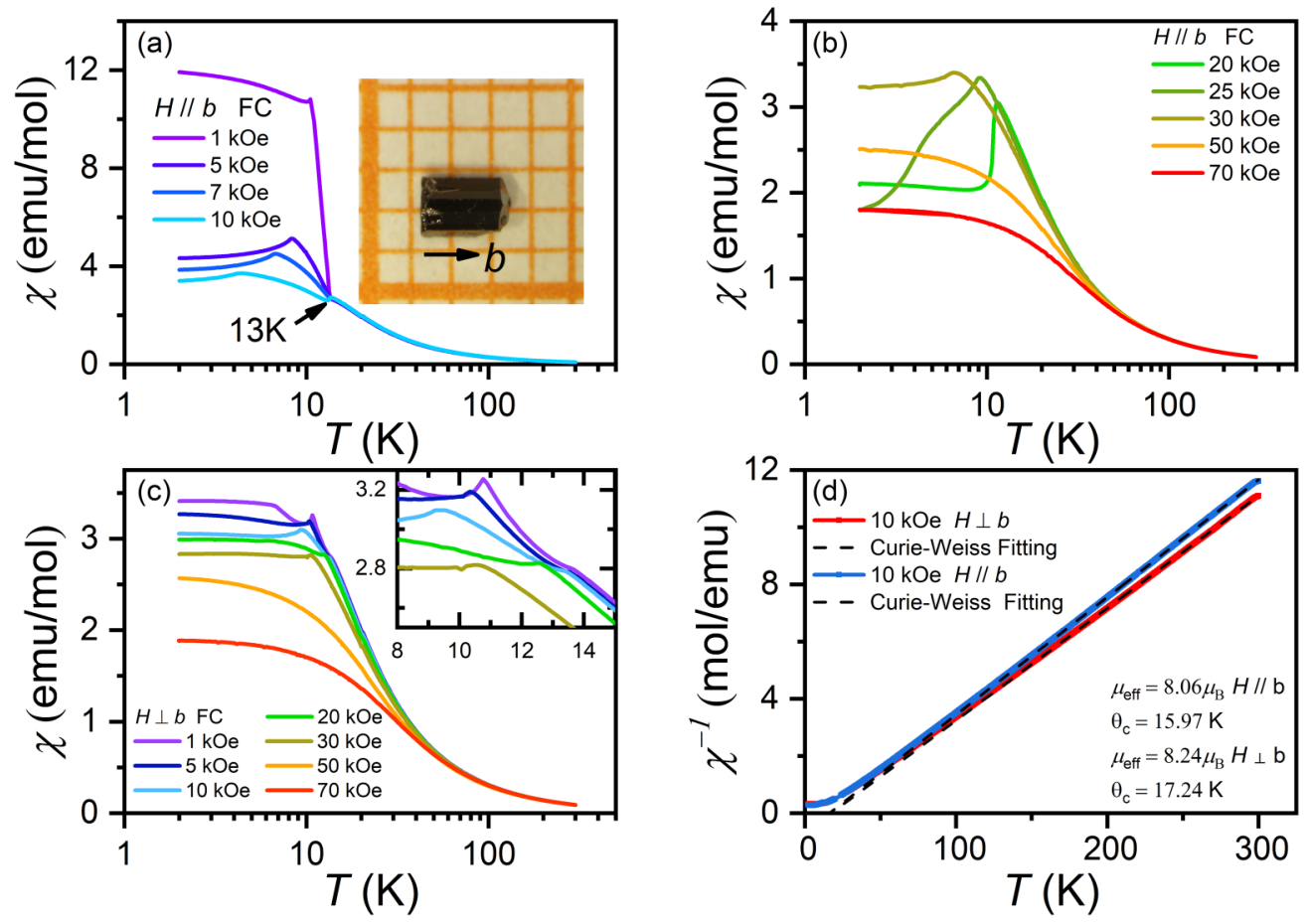

FIG. 4. (a) and (b) Temperature dependence of magnetic susceptibility for $H \| b$ with varied magnetic fields. The inset in (a) shows the single crystal used for measurements. (c) Temperature dependence of magnetic susceptibility for $H \perp b$ with varied magnetic fields. The inset plots an enlarged view for temperature ranges of 8-15 K. (d) Temperature dependence of the inverse magnetic susceptibility and Curie-Weiss fitting.

to $1.7 \mu_{B} /$ f.u. with the magnetic field increasing from 0 to 183 Oe. The magnetizations then increase linearly with the increase in magnetic fields up to $12.6 \mathrm{kOe}$ and reach a plateau of about $7.1 \mu_{B}$ /f.u. The magnetizations increase sharply to

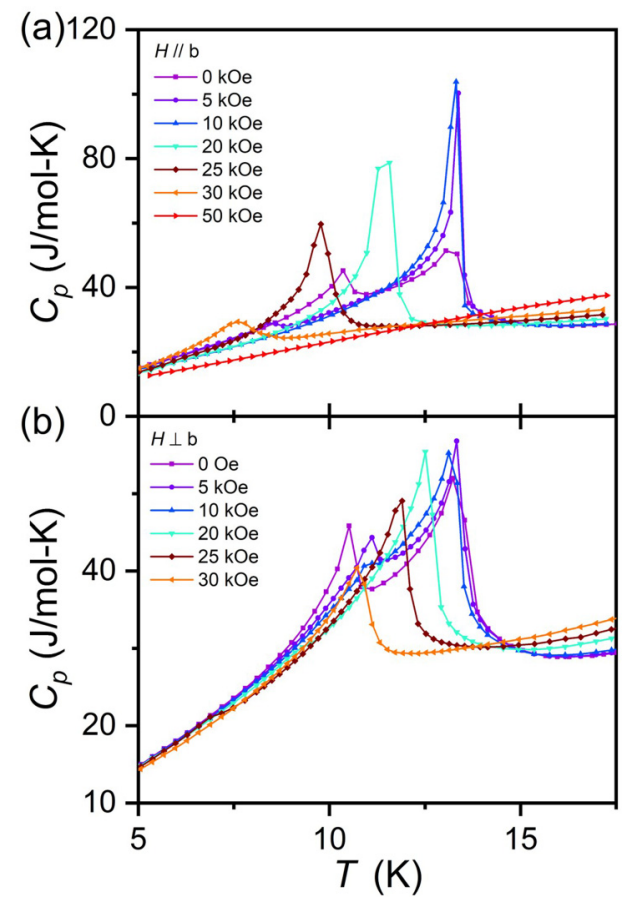

FIG. 5. (a) and (b) Temperature dependence of specific heat of $\mathrm{Eu}_{3} \mathrm{InAs}_{3}$ measured with $H \| b$ and $H \perp b$, respectively.

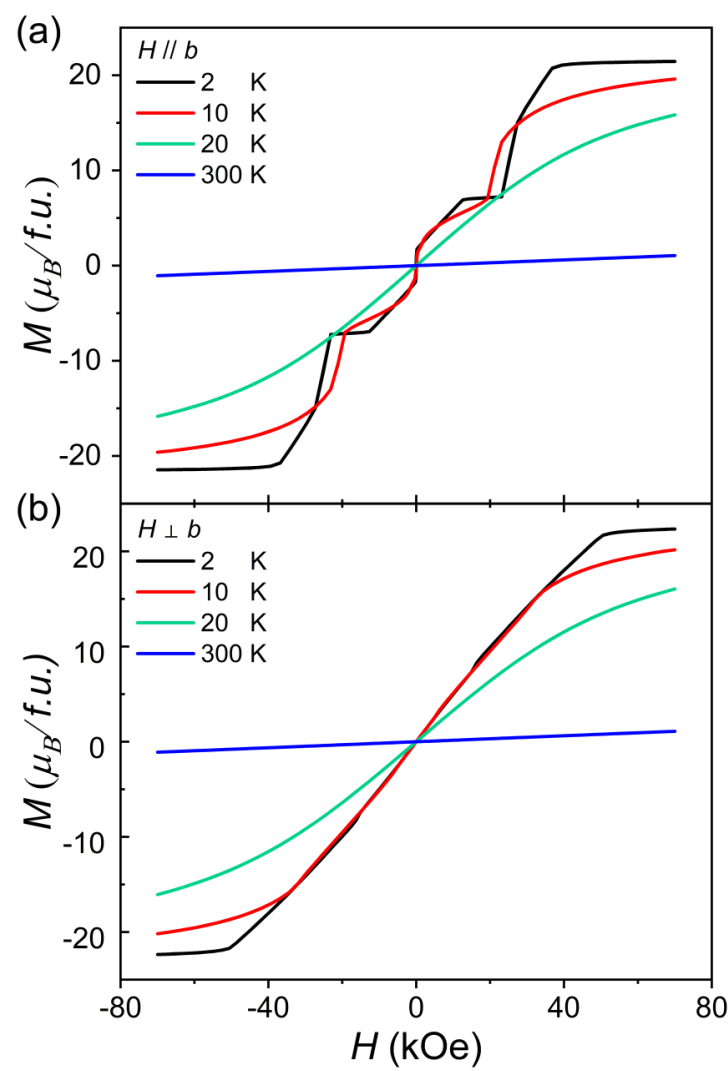

FIG. 6. (a) and (b) The isothermal magnetization curves of $\mathrm{Eu}_{3} \mathrm{InAs}_{3}$ for $H \| b$ and $H \perp b$, respectively. 

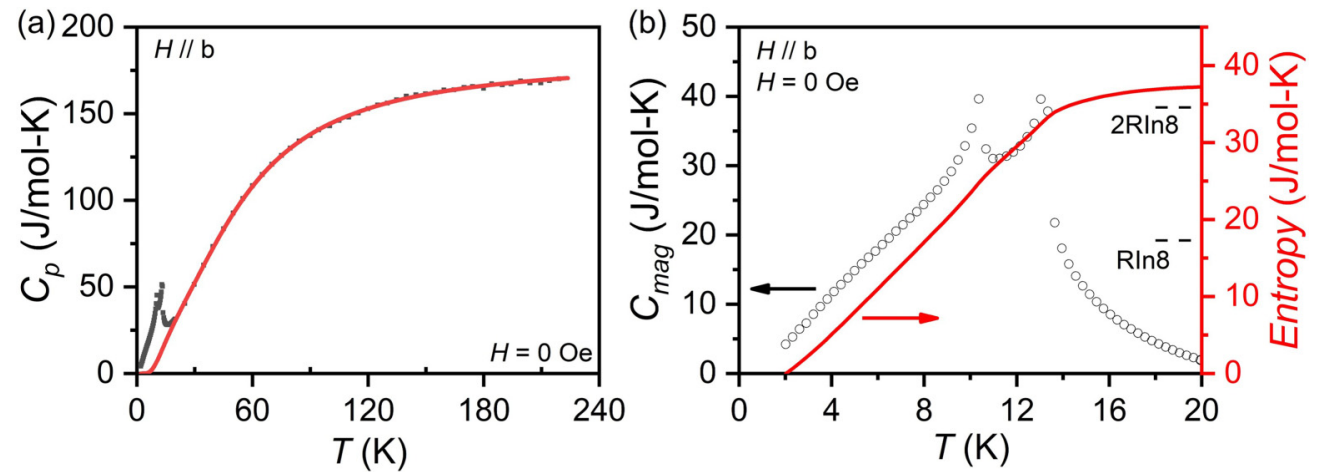

FIG. 7. (a) Temperature dependence of specific heat of $\mathrm{Eu}_{3} \operatorname{InAs}_{3}$ measured without a magnetic field, and the red curve indicates the Debye-Einstein fitting. (b) The magnetic contributions of the specific heat and the corresponding entropy.

$14.4 \mu_{B} /$ f.u. when $H$ increases from 23.2 to $26.6 \mathrm{kOe}$, then the magnetizations increase less sharply and are nearly saturate (22.2 $\mu_{B}$ /f.u.) when $H$ is larger than $37.0 \mathrm{kOe}$. The $M_{\mathrm{S}}$ of $22.2 \mu_{B} /$ f.u. is consistent with the moments of three paralleled $\mathrm{Eu}^{2+}$ ions, indicating a field-induced ferromagnetic state with all $\mathrm{Eu}^{2+}$ moments are parallel arranged. It should be noted that the values of $7.0 \mu_{B}$ /f.u. (the plateau) and $14.4 \mu_{B} /$ f.u. (the slope change) are close to $1 / 3$ and $2 / 3$ of the $M_{\mathrm{S}}$ for $\mathrm{Eu}_{3} \mathrm{InAs}_{3}$. At $10 \mathrm{~K}$, the $M(H)$ curve does not show the plateau but only anomalies around $1 / 3$ and $2 / 3$ of $M_{\mathrm{S}}$. The $M(H)$ curve measured at $20 \mathrm{~K}$ is smooth and is not saturated under $70 \mathrm{kOe}$. The $M(H)$ curve measured at $300 \mathrm{~K}$ is linear and is following the paramagnetic behavior. The phenomenon of observation of fractional values of $M_{\mathrm{S}}$ is usually related to the geometric frustration resulted from triangular lattices or competition of exchange interactions [32]. The ground state of $\mathrm{Eu}_{3} \mathrm{InAs}_{3}$ is $\mathrm{AFM}$, but positive $\theta_{\mathrm{W}}$ values indicate that ferromagnetic interactions are dominant in $\mathrm{Eu}_{3} \mathrm{InAs}_{3}$. One possible reason for the emergence of the plateau at $1 / 3$ of $M_{\mathrm{S}}$ is due to the competing AFM and ferromagnetic exchange interactions in $\mathrm{Eu}_{3} \mathrm{InAs}_{3}$. Moreover, the $\mathrm{Eu}_{1}, \mathrm{Eu}_{2}$, and $\mathrm{Eu}_{3}$ atoms can form a pseudotriangular lattice in $\mathrm{Eu}_{3} \mathrm{InAs}_{3}$ [see Fig. 1(b)], which may result in a kind of frustration.

Figure 6(b) shows the $M(H)$ curves of $E_{3} I_{n} A_{s_{3}}$ measured with $H \perp b$. At $2 \mathrm{~K}$, the magnetizations increase linearly with the increase in the magnetic fields and are nearly saturated (21.5 $\mu_{B} /$ f.u) when the magnetic fields reach $50 \mathrm{kOe}$, indicating a field-induced ferromagnetic state. The $M(H)$ curves measured at 10 and $20 \mathrm{~K}$ are simila,r but the magnetizations are not saturated at $70 \mathrm{kOe}$. Paramagnetic behavior is observed at $300 \mathrm{~K}$.

The $C_{p}(T)$ curve without the magnetic field for $\mathrm{Eu}_{3} \mathrm{InAs}_{3}$ up to $270 \mathrm{~K}$ is shown in Fig. 7(a). To estimate the specific contribution from a phonon and an electron, the data between 50 and $200 \mathrm{~K}$ are fitted with the Debye-Einstein model,

$$
\begin{aligned}
C_{p}= & \lambda T+9 n R \alpha\left(\frac{T}{T_{D}}\right)^{3} \int_{0}^{T_{p} / T} \frac{x^{4} e^{x}}{\left(e^{x}-1\right)^{2}} d x \\
& +\frac{3 n R(1-\alpha) e^{T_{E} / T}}{\left(e^{T_{E} / T}-1\right)^{2}},
\end{aligned}
$$

where $C_{p}, \lambda, n, R$, and $\alpha$ represent the isobaric specific heat, the electronic specific heat, the moles, the Boltzmann constant, and the weight of different contributions, respectively.
The $T_{E}$ and $T_{D}$ represent Einstein and Debye temperatures, respectively. The fitting curve is shown in Fig. 7(a), and the resulting values for $T_{D}$ and $\gamma$ are $239 \mathrm{~K}$ and $15 \mathrm{mJmol}^{-1} \mathrm{~K}^{-2}$, respectively. The corresponding magnetic entropy is calculated by subtracting the phonon and electron contributions [see Fig. 7(b)]. The calculated entropy is larger than $2 R \ln 8$ but is smaller than $3 R \ln 8$ (expected for three $\mathrm{Eu}^{2+}$ ions), where $R$ is the gas constant. The small deviation from $3 R \ln 8$ may indicate that the fitted phonon and electron contributions are overestimated.

\section{CONCLUSION}

Single crystals of $\mathrm{Eu}_{3} \mathrm{InAs}_{3}$ have been successfully synthesized by indium flux reactions. SCXRD analysis indicates that $\mathrm{Eu}_{3} \mathrm{InAs}_{3}$ crystallizes in an orthorhombic space-group Pnma with cell parameters, $a=12.8973(4), b=4.39530(10) \AA$, and $c=13.9273(5) \AA$, which are close to the reported values [21]. Theoretical calculations indicate that $\mathrm{Eu}_{3} \operatorname{InAs}_{3}$ is a semiconductor with a direct band gap of $0.3 \mathrm{eV}$. Measurements of Seebeck coefficients found that $\mathrm{Eu}_{3} \mathrm{InAs}_{3}$ has large negative Seebeck coefficients similar to that reported in Refs. [9,21]. Currently, the as-grown samples have relatively high electrical resistivity, indicating a quite low intrinsic carrier concentration. Future doping studies are needed to optimize the thermoelectric properties of $\mathrm{Eu}_{3} \mathrm{InAs}_{3}$.

Anisotropic magnetic properties are measured for both $H \| b$ and $H \perp b$, respectively. Temperature-dependent magnetic susceptibility and specific-heat measurements confirm two AFM transitions for both $H \| b\left(T_{N 1 \|}=13\right.$ and $T_{N 2 \|}=$ $10 \mathrm{~K})$ and $H \perp b\left(T_{N 1 \perp}\right)=13$ and $\left.T_{N 2 \perp}=10 \mathrm{~K}\right)$. Increasing magnetic field suppresses $T_{N 2}$ first then $T_{N 1}$ for both $H \| b$ and $H \perp b$. Isothermal magnetization measurements at $2 \mathrm{~K}$ with $H \| b$ indicate successive field-induced metamagnetic transitions, particularly, the magnetization curves show a plateau at $1 / 3$ of $M_{\mathrm{S}}$ and an anomaly at $2 / 3$ of $M_{\mathrm{S}}$ before reaching $M_{\mathrm{S}}$. In comparison, isothermal magnetizations at $2 \mathrm{~K}$ with $H \perp b$ increase linearly with the increase in magnetic fields and nearly saturate when the magnetic fields are larger than $50 \mathrm{kOe}$. The origin of the plateau at $1 / 3$ of $M_{\mathrm{S}}$ observed in $\mathrm{Eu}_{3} \mathrm{InAs}_{3}$ may be related to competing AFM and ferromagnetic exchange interactions in $\mathrm{Eu}_{3} \mathrm{InAs}_{3}$. Future studies, such as neutron diffraction to determine magnetic structures under varied magnetic fields, are needed to understand it. $\mathrm{Eu}_{3} \mathrm{InAs}_{3}$ is a Zintl phase showing a 1/3 magnetization plateau. 


\section{ACKNOWLEDGMENTS}

This work was supported by the Beijing Natural Science Foundation (Grant No. Z180008) and the National Natural Science Foundation of China (Grants No. 12104492, No. 52172259, and No. U2032204), National Key Re- search and Development Program of China (Grants No. 2017YFA0302900 and No. 2018YFA0702100), the Strategic Priority Research Program of the Chinese Academy of Sciences (Grant No. XDB33010000), and the K. C. Wong Education Foundation (Grant No. GJTD-2018-01).
[1] E. Zintl and W. Dallenkopf, Über den Gitterbau von NaTl und seine Beziehung zu den Strukturen vom Typus des $\beta$-Messings, Z. Phys. Chem. 16B, 195 (1932).

[2] H. Auer, R. Guehne, M. Bertmer, S. Weber, P. Wenderoth, T. C. Hansen, J. Haase, and H. Kohlmann, Hydrides of alkaline earth-tetrel (AeTt) Zintl phases: Covalent $\mathrm{Tt}-\mathrm{H}$ bonds from silicon to tin, Inorg. Chem. 56, 1061 (2017).

[3] K.-F. Liu and S.-Q. Xia, Recent progresses on thermoelectric Zintl Phases: Structures, materials and optimization, J. Solid State Chem. 270, 252 (2019).

[4] J. Shuai, J. Mao, S. Song, Q. Zhang, G. Chen, and Z. Ren, Recent progress and future challenges on thermoelectric Zintl materials, Mater. Today Physics 1, 74 (2017).

[5] S. M. Kauzlarich, S. R. Brown, and G. Jeffrey Snyder, Zintl phases for thermoelectric devices, Dalton Trans. 20072099.

[6] G. Cordier, H. Schäfer, and M. Stelter, $\mathrm{Sr}_{3} \mathrm{GaSb}_{3}$ and $\mathrm{Sr}_{3} \mathrm{InP}_{3}$, Two new Zintl phases with complex anions, Z. Naturforsch. B 42, 1268 (1987).

[7] S. Stoyko, L. Voss, H. He, and S. Bobev, Synthesis, crystal and electronic structures of the pnictides $\mathrm{AE}_{3} \operatorname{TrPn}_{3}(\mathrm{AE}=\mathrm{Sr}, \mathrm{Ba}$; $\operatorname{Tr}=\mathrm{Al}, \mathrm{Ga} ; \mathrm{Pn}=\mathrm{P}, \mathrm{As})$, Crystals 5, 433 (2015).

[8] Q. Shi, Z. Feng, Y. Yan, and Y. X. Wang, Electronic structure and thermoelectric properties of Zintl compounds $\mathrm{A}_{3} \mathrm{AlSb}_{3}(\mathrm{~A}=\mathrm{Ca}$ and $\mathrm{Sr})$ : First-principles study, RSC Adv. 5, 65133 (2015).

[9] N. Kazem, A. Hurtado, F. Sui, S. Ohno, A. Zevalkink, J. G. Snyder, and S. M. Kauzlarich, High temperature thermoelectric properties of the solid-solution Zintl phase $\mathrm{Eu}_{11} \mathrm{Cd}_{6-x} \mathrm{Zn}_{\mathrm{x}} \mathrm{Sb}_{12}$, Chem. Mater. 27, 4413 (2015).

[10] W. Zhang, C. Chen, H. Yao, W. Xue, S. Li, F. Bai, Y. Huang, X. Li, X. Lin, F. Cao, J. Sui, S. Wang, B. Yu, Y. Wang, X. Liu, and Q. Zhang, Promising Zintl-phase thermoelectric compound SrAgSb, Chem. Mater. 32, 6983 (2020).

[11] E. S. Toberer, A. Zevalkink, N. Crisosto, and G. J. Snyder, The Zintl compound $\mathrm{Ca}_{5} \mathrm{Al}_{2} \mathrm{Sb}_{6}$ for low-cost thermoelectric power generation, Adv. Funct. Mater. 20, 4375 (2010).

[12] A. Zevalkink, G. Pomrehn, Y. Takagiwa, J. Swallow, and G. J. Snyder, Thermoelectric properties and electronic structure of the Zintl-phase $\mathrm{Sr}_{3} \mathrm{AlSb}_{3}$, ChemSusChem 6, 2316 (2013).

[13] A. Zevalkink, W. G. Zeier, G. Pomrehn, E. Schechtel, W. Tremel, and G. J. Snyder, Thermoelectric properties of $\mathrm{Sr}_{3} \mathrm{GaSb}_{3}$ - a chain-forming Zintl compound, Energy Environ. Sci. 5, 9121 (2012).

[14] K. Shinozaki, Y. Goto, K. Hoshi, R. Kiyama, N. Nakamura, A. Miura, C. Moriyoshi, Y. Kuroiwa, H. Usui, and Y. Mizuguchi, Thermoelectric properties of the As/P-based Zintl compounds $\mathrm{EuIn}_{2} \mathrm{As}_{2-x} \mathrm{P}_{\mathrm{x}}(X=0$ to 2$)$ and $\mathrm{SrSn}_{2} \mathrm{As}_{2}$, ACS Appl. Energy Mater. 4, 5155 (2021).

[15] A. Balvanz, J. Qu, S. Baranets, E. Ertekin, P. Gorai, and S. Bobev, New N-Type Zintl phases for thermoelectrics: Discovery, structural characterization, and band engineering of the compounds $\mathrm{A}_{2} \mathrm{CdP}_{2}(\mathrm{~A}=\mathrm{Sr}, \mathrm{Ba}, \mathrm{Eu})$, Chem. Mater. 32, 10697 (2020).

[16] J. Jiang, A. C. Payne, M. M. Olmstead, H. Lee, P. Klavins, Z. Fisk, S. M. Kauzlarich, R. P. Hermann, F. Grandjean, and G. J. Long, Complex magnetic ordering in $\mathrm{Eu}_{3} \mathrm{InP}_{3}$ : A new rare earth metal Zintl compound, Inorg. Chem. 44, 2189 (2005).

[17] T. Chatterji and W. Henggeler, MSR investigation of the magnetic ordering in $\mathrm{EuAs}_{3}$, Solid State Commun. 132, 617 (2004).

[18] J. Blawat, P. Swatek, X. Gui, R. Jin, and W. Xie, Antiferromagnetic semiconductor $\mathrm{Eu}_{3} \mathrm{Sn}_{2} \mathrm{P}_{4}$ with $\mathrm{Sn}-\mathrm{Sn}$ dimer and crown-wrapped Eu, J. Mater. Chem. C 7, 12650 (2019).

[19] J. Y. Chan, M. E. Wang, A. Rehr, S. M. Kauzlarich, and D. J. Webb, Synthesis, structure, and magnetic properties of the rareearth Zintl compounds $\mathrm{Eu}_{14} \mathrm{MnPn}_{11}$ and $\mathrm{Eu}_{14} \operatorname{InPn}_{11}(\mathrm{Pn}=\mathrm{Sb}$, Bi), Chem. Mater. 9, 2131 (1997).

[20] M. Q. Arguilla, N. D. Cultrara, Z. J. Baum, S. Jiang, R. D. Ross, and J. E. Goldberger, $\mathrm{EuSn}_{2} \mathrm{As}_{2}$ : An exfoliatable magnetic layered Zintl-Klemm phase, Inorg. Chem. Front. 4, 378 (2017).

[21] K. Rajput, S. Baranets, and S. Bobev, Observation of an unexpected n-type semiconducting behavior in the new ternary Zintl phase $\mathrm{Eu}_{3} \mathrm{InAs}_{3}$, Chem. Mater. 32, 9616 (2020).

[22] I. Schellenberg, M. Eul, C. Schwickert, C. M. Kubata, E. Cuervo Reyes, R. Nesper, U. Ch. Rodewald, and R. Pöttgen, The Zintl phases $\mathrm{Eu}_{3} \mathrm{Mg}_{5} \mathrm{Si}_{5}$ and $\mathrm{Eu}_{3} \mathrm{Mg}_{5} \mathrm{Ge}_{5}$, Z. Anorg. Allg. Chem. 638, 1976 (2012).

[23] W. Bauhofer, T. Chattopadhyay, M. Möllendorf, E. Gmelin, H. G. von Schnering, U. Steigenberger, and P. J. Brown, magnetic phase diagram of $\mathrm{EuAs}_{3}$, J. Magn. Magn. Mater. 54-57, 1359 (1986).

[24] J. V. Badding and A. M. Stacy, Magnetic phase transitions in $\mathrm{EuNi}_{5} \mathrm{P}_{3}$ : Unusual steps in the magnetization with field, Phys. Rev. B 35, 8880 (1987).

[25] J. Jiang, M. M. Olmstead, S. M. Kauzlarich, H.-O. Lee, P. Klavins, and Z. Fisk, Negative magnetoresistance in a magnetic semiconducting Zintl phase: $\mathrm{Eu}_{3} \mathrm{In}_{2} \mathrm{P}_{4}$, Inorg. Chem. 44, 5322 (2005).

[26] S. Sarkar, M. J. Gutmann, and S. C. Peter, The crystal structure

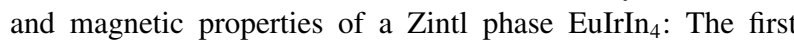
member of the Eu-Ir-in family, Dalton Trans. 43, 15879 (2014).

[27] M. Radzieowski, F. Stegemann, S. Klenner, Y. Zhang, B. P. T. Fokwa, and O. Janka, On the divalent character of the eu atoms in the ternary Zintl phases $\mathrm{Eu}_{5} \mathrm{In}_{2} \mathrm{Pn}_{6}$ and $\mathrm{Eu}_{3} \mathrm{MAs}_{3}(\mathrm{Pn}=$ As-Bi; $\mathrm{M}=\mathrm{Al}, \mathrm{Ga}$ ), Mater. Chem. Front. 4, 1231 (2020).

[28] T. F. Fässler and C. Kronseder, $\mathrm{BaSn}_{3}$ : A Superconductor at the border of Zintl phases and intermetallic compounds. Real-space analysis of band structures, Angew. Chem., Int. Ed. Engl. 36, 2683 (1997).

[29] J. L. Mathieu and S. E. Latturner, Zintl phase as dopant source in the flux synthesis of $\mathrm{Ba}_{1-x} \mathrm{~K}_{\mathrm{x}} \mathrm{Fe}_{2} \mathrm{As}_{2}$ type superconductors, Chem. Commun. 2009, 4965. 
[30] L. Deakin, R. Lam, F. Marsiglio, and A. Mar, Superconductivity in $\mathrm{Ba}_{2} \mathrm{Sn}_{3} \mathrm{Sb}_{6}$ and $\mathrm{SrSn}_{3} \mathrm{Sb}_{4}$, J. Alloys Compd. 338, 69 (2002).

[31] Supplemental Material at http://link.aps.org/supplemental/10. 1103/PhysRevResearch.3.043178 for the crystallographic in- formation file of $\mathrm{Eu}_{3} \mathrm{InAs}_{3}$ obtained from the refinement of the single-crystal x-ray-diffraction data.

[32] B. Schmidt and P. Thalmeier, Frustrated two dimensional quantum magnets, Phys. Rep. 703, 1 (2017). 